\title{
Centrality and reciprocity in directed social networks - A case study
}

\author{
Deepa V.G. ${ }^{1 *}$, Aparna Lakshmanan S. ${ }^{2}$, and Sreeja V. N. ${ }^{3}$
}

\begin{abstract}
The idea of Social Network is to map social relations and to look for parameters which will eventually lead to a better understanding of how relations work, information flows and organizations collaborate. In this paper, the data collected from a tribal village regarding their interaction (which includes advisory help, monitory help and companionship during leisure time) during normal and crisis periods have been represented as a directed graph. This graph is used to identify most influential members in the tribal community and steps to be taken for upliftment of the society.
\end{abstract}

\section{Keywords}

Social Network, SNA, Reciprocity, Centrality, Tribal study, Malayan.

\section{AMS Subject Classification}

91D30, 91D10, 05C82.

1,3 Department of Mathematics, Sree Krishna College, Guruvayur- 680102, Kerala, India.

${ }^{2}$ Department of Mathematics, St. Xavier's College for Women, Aluva-683101, Kerala, India.

*Corresponding author: ${ }^{1}$ skcdeepa@gmail.com; ${ }^{2}$ aparnaren@gmail.com; ${ }^{3}$ drsreejavn@gmail.com

Article History: Received 24 January 2019; Accepted 24 May 2019

(C)2019 MJM.

\section{Contents}

1 Introduction 479

1.1 Objectives and Main Contributions . . . . . 480

2 Social Network Data. .480

2.1 Results and Discussion . . . . . . . . . . 482

3 Conclusion 483

References 484

\section{Introduction}

Network Analysis is a growing field with a great deal of opportunities. Despite this popularity, there exist considerable confusion about network theory. In the study of literature, Network analysis has been applied by Anheier, Gerhards and Romo [7] and Wouter De Nooy [13]. One who is interested in classic network methods may refer Wasserman and Faust [12] and Brandes and Erlebach [1]. Some more recent developments can be found in Carringtom, Scott and Wasserman [10]. Social Network (SN) refers to the articulation of a social relationship, ascribed or achieved, among individuals, families, households, villages, communities, religions and so on. The original concept of Social Circles was introduced by Georg Simmel, at the beginning of the 20th century. SN has its origin in Mathematics (Graph theory) and Sociology, but now it is being used across a wide range of other disciplines.

Leonard Paul Euler (1707-1783) was a pioneering Swiss Mathematician who led the foundation of very vast field of Mathematics, the multidisciplinary area graph theory by solving the 'Koningsberg bridge' problem (1736) originated in the city of Koningsberg, formerly in Germany, now a part of Russia. Even though the field originated in 1736, it took 200 years to write a book on graph theory. Since then graph theory has developed in to an extensive and popular branch of Mathematics, which has been applied to many practical problems in Mathematics, Computer Science and other scientific and non-scientific areas. In many cases, the standard models from graph theory may not be suitable, in such cases a better option will be social network analysis.

Social Network Analysis (SNA) is the process of investigating social structures through the use of Network and Graph Theory. SN perspective focuses on relationship among social entities (actor). Examples of social structures include social media networks, friendship and acquaintance network, collaboration graphs, kinship, disease transmission and sexual relationship. SNA is not tied to a specific theory of how society or individuals function. When considering its use 
for evaluation purposes, since each program or project will normally has its own theory of change, SNA might be best represented as a representational technology. The three main aspects of this technology are Network Diagrams, Network Matrices and Mathematical Measures describing the structure of networks, and place of actors (individuals, groups etc.) within them. $\mathrm{SN}$ is a tool that describes the association between the units of interest. The spread of personal computer usage has encouraged wider use of SNA methods because it has increased ability to manage large data sets and to visualize SN data in a wide variety of ways.

SNA has its theoretical roots in Sociology. Georg Simmel and Emile Durkheim [5] developed the importance of studying patterns of relationships between individuals. Social-culturaleconomical-emotional-intellectual concepts sometimes fails to express using natural language. Therefore, the scientist in Social Network may seek help from other disciplines. The best lender is graph theory, the branch of Mathematics which is concerned with discrete relational structures. The close relationship between graph theory and the study of social networks is much like the relationship between Physical Science and Differentiation: in both cases the mathematical literature provides a formal substrate for the associated scientific work. Here we consider network structure in terms of vertex or nodes (individual actor or a group of people) and the ties, edges or links (relationships or interactions) that connect them. If these nodes are represented as points and ties are represented as directed lines, we can visualize these network as sociograms. So SNA provide a powerful tool to analyse several types of relationship among individuals or a group of individuals in $21^{\text {st }}$ century.

\subsection{Objectives and Main Contributions}

This paper contributes to our understanding of a tribal colony in India from the $\mathrm{SN}$ perspective by measuring their interaction (which includes advisory help, monitory help and companionship during leisure time) during normal and crisis period. In addition, this paper enquires the number of members, educational qualification and age of the head of the house hold in each family.

\section{Social Network Data}

Elanad (Coordinates: $10^{0} 37^{\prime} 0^{\prime \prime} N 76^{0} 23^{\prime} 0^{\prime \prime} E$ ) is a village in Pazhayannur panchayathu, Thrissur District in the state of Kerala, India with a population 11376 [9]. .

Malayan (Details taken from the web site http://www.foci kerala.org) belongs to Scheduled Tribe in Kerala (as amended by the Scheduled Castes and Scheduled Tribes Order (Amendment Act) 1976 and as amended by the Constitution (Scheduled castes) Orders (Second Amendment) Act, 2002 (Act 61 of 2002) vide Part VIII- Kerala- Schedule I notified in the Gazette of India, dated 18 December, 2002 and as amended by

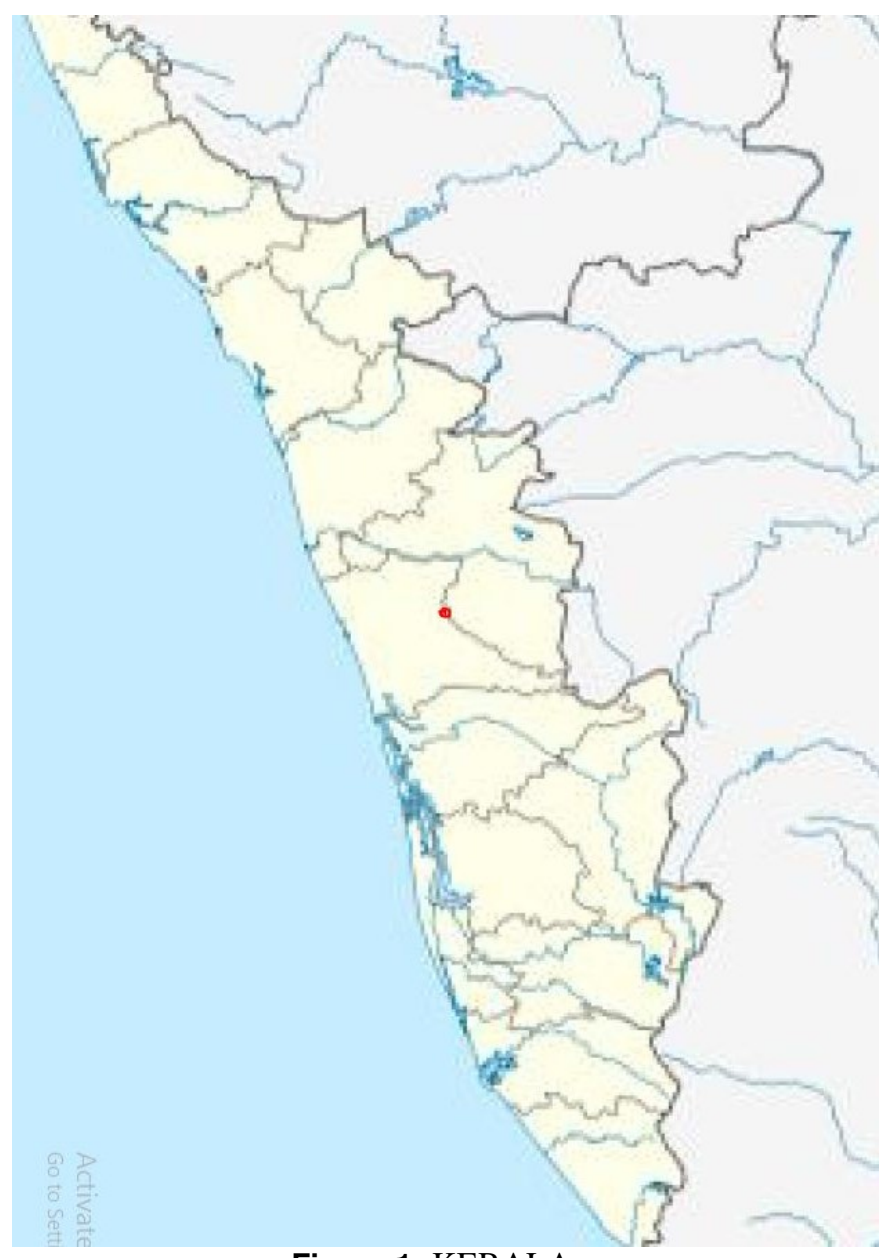

Figure 1. KERALA

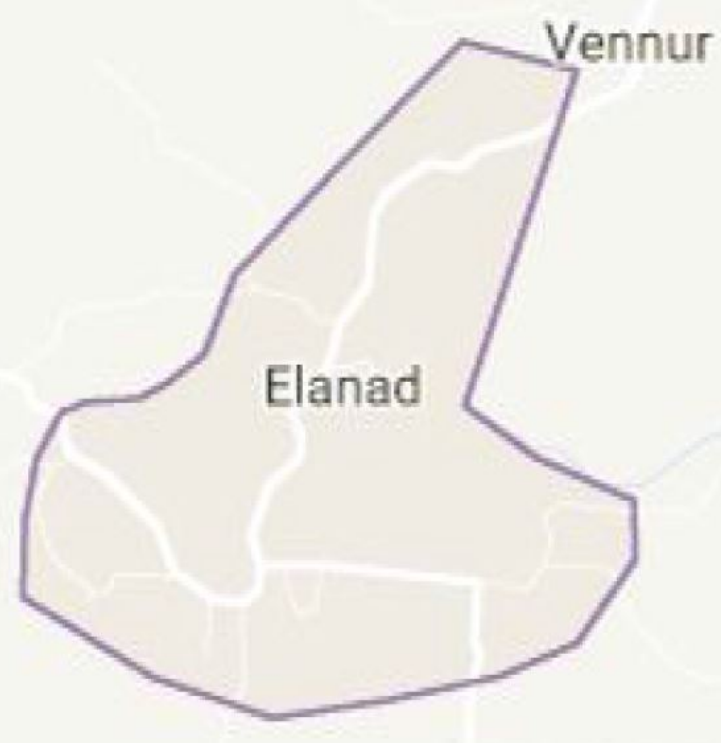

Figure 2. ELANAD LOCATION 
the Scheduled Castes and Scheduled Tribes Orders (Amendment) Act 2002 (Act 10 of 2003) vide Part VII- Kerala- Second Schedule notified in the Gazette of India dated 8 January, 2003). The Malayans are mainly found in the hilly regions of Palakkad and Thrissur districts of Kerala. As per sensus 2011, 118 Scheduled tribes belonging to Malayan caste lives in ward 10, Elanad Village, Kerala, India. Most of them at present are agricultural workers and some of them are involved in collecting forest produce.

The population of our social network study is 39 houses in the tribal village Thirumani of Elanad in Kerala, India. We have collected data through a questionnaire and personal interview. The 39 houses are labeled with the numbers $1,2, \ldots, 39$; for each house $i$ we have data consisting of the list of houses whom they interact (for companionship for spending leisure time, seeking advice and monitory help) during normal and crisis period. The data gives directed graph $D=(V, E)$ where the vertex set $V$ is the set of 39 houses and $(u, v)$ is an arc in $E$ if $u$ approaches $v$. The resulting directed graph is given in Figure 3 and Figure 4.

The network diagram of normal period is a directed graph with 39 nodes and 27 ties and that of during the crisis period is a complete bipartite graph $K_{1,38}$ (star).

The primary form of data storage for social network analysis is the sociomatrix (adjacency matrix). It is the tabular representation in matrix form of data collected using a sociomatrix method to measure interpersonal relationships. There is one column and one raw for each actor. It is an $n \times n$ square matrix (where cardinality of $V$ is $n$ ) whose $i j^{t h}$ cell is equal to 1 if vertex $i$ sends an edge to vertex $j$ and 0 otherwise. For an undirected network with Adjacency matrix $A$, it is clear that $A_{i j}=A_{j i}$, ie; $A$ must be symmetric. This is not the case in directed network. If the network under consideration is simple, the diagonal elements of the sociomatrix is identically zero. Otherwise, $A_{i i}=1$ if and only if vertex $i$ has a loop (For both directed and undirected networks).

The degree centrality is the most basic network measure and captures the number of ties to a given actor. For undirected ties, this is simply a number of ties for every actor. For directed networks, actors can have both in-degree and out-degree centrality scores. Centrality measures, how central or well connected an actor is in a network. This theoretically signals importance or power and increased access to information, or general activity level.

The in-degree of a vertex $v$ is

$N^{+}(v)=\mid\{i \in E:$ the edge $(i, v) \in E(G)\} \mid$

and

the out-degree of a vertex $v$ is

$N^{-}(v)=\mid\{i \in E:$ the edge $(v, i) \in E(G)\} \mid$.

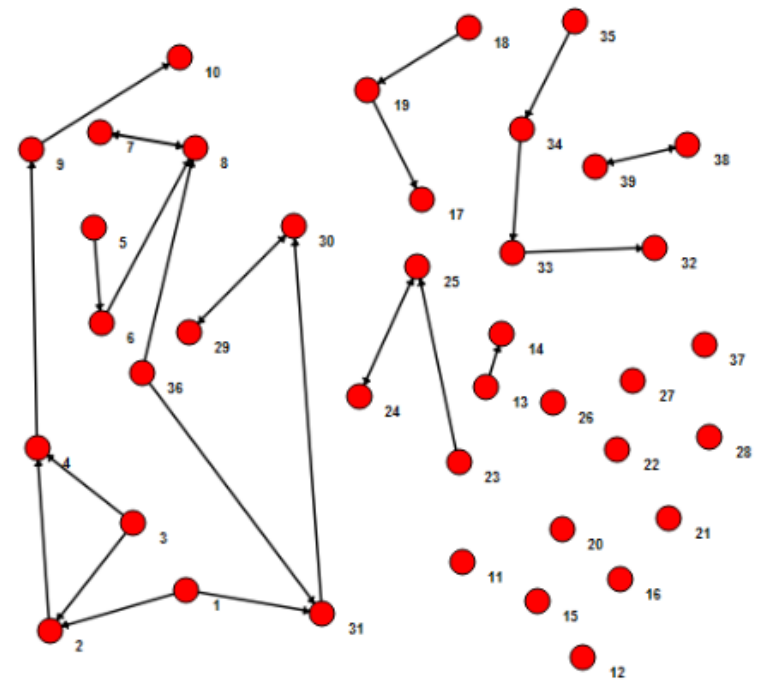

Figure 3. INTERACTION- NORMAL PERIOD

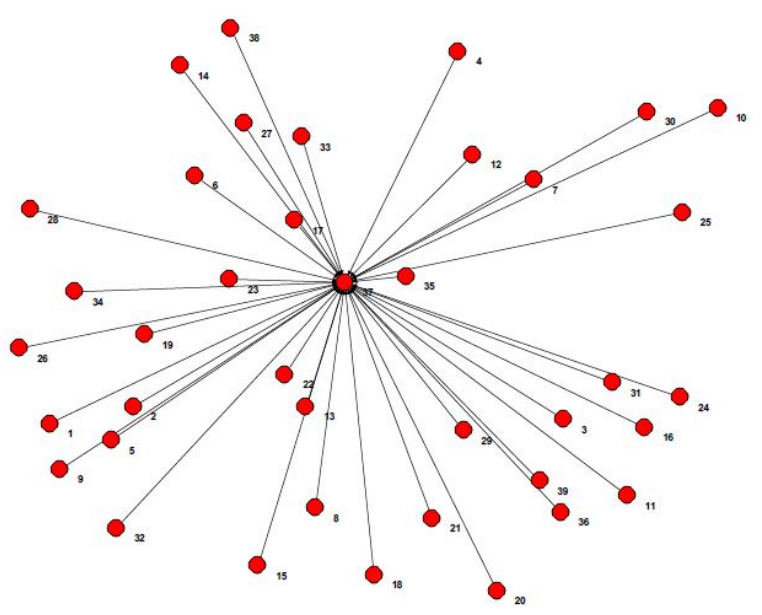

Figure 4. INTERACTION-CRISIS PERIOD 
If $N^{+}(v)=N^{-}(v)=0$ then $v$ is isolated or independent; $N^{+}(v)=0, N^{-}(v)>0$, then $v$ is weak in society;

$N^{+}(v)>0, N^{-}(v)=0$ then $v$ is dominant or decision making and

$N^{+}(v)>0, N^{-}(v)>0$ then $v$ is linkage, adjustable or dependent.

Reciprocity refers to responding to a positive action with another positive action; it creates maintains and strengthens various social bounds [11]. Reciprocity is a form of social obligation and is a motivation for returning favours received from others. In simple, it is a number which gives the extend to which support is both given and received in a relationship. The concept of reciprocity was introduced in the beginning of SNA. Reciprocity is the tendency for directed ties from actor $i$ to actor $j$ be reciprocated and send back from actor $j$ to actor $i$. This captures the classic finding that feeling and actions tend to be reciprocated. If reciprocity is equal to zero, it is uni-directional and if it is one, it is bidirectional.

\subsection{Results and Discussion}

During normal period, it seems that 11 families are isolated. When we look in to it, it is found that most of this houses are in boundary. The members of this family used to do work in nearby houses. Hence they possess Stronger ties with these houses and normally interacts with them during normal time. It is also noted that majority likes to interact with either those people who have blood relation with them or live adjacent to them. The Maximal connected subgraph contains 14 actors.

The sociomatrix of our network data is a $39 \times 39$ square matrix. The sociomatrix of both normal and crisis period are given below.

When we go through the sociomatrix of normal period, even though there may be 1521 entries it seems that nearly 1.78 percentage elements are unity (the network under consideration is loop less, the diagonal elements are identically zero). This indicates most of the families does not approach a mentor (advisory help, monitory help and companionship during leisure time) during normal period. This may be due to either they have the capacity to take decisions independently or due to the lack of sources providing support and helping mentality.

The sociomatrix during crisis period invites attention to the coloumn corresponding to the actor 37 which is identically one (except at $37^{\text {th }}$ entry, since the network is loop less). All other entries are zero. This is due to the hereditable structure, the one and only one important character in the colony is, so called 'mooppan'. He is an elected person by 'samudayam' which includes the secretary of the local body, sc promoter, ward member etc. All the families interacted to the external body through the mooppan. In case of emergency, all of them (they are financially stable or not) approaches mooppan and receive advises from him. If he can not take decision indepen-

\section{SOCIOMATRLX DURING NORMAL PERIOD}

010000000000000000000000000000100000000 000100000000000000000000000000000000000 010100000000000000000000000000000000000 000000001000000000000000000000000000000 000001000000000000000000000000000000000 000000010000000000000000000000000000000 000000010000000000000000000000000000000 000000100000000000000000000000000000000 000000000100000000000000000000000000000 000000000000000000000000000000000000000 000000000000000000000000000000000000000 000000000000000000000000000000000000000 000000000000010000000000000000000000000 000000000000000000000000000000000000000 000000000000000000000000000000000000000 000000000000000000000000000000000000000 000000000000000000000000000000000000000 000000000000000000100000000000000000000 000000000000000010000000000000000000000 000000000000000000000000000000000000000 000000000000000000000000000000000000000 000000000000000000000000000000000000000 000000000000000000000000100000000000000 000000000000000000000000100000000000000 000000000000000000000001000000000000000 000000000000000000000000000000000000000 000000000000000000000000000000000000000 000000000000000000000000000000000000000 000000000000000000000000000001000000000 000000000000000000000000000010000000000 000000000000000000000000000001000000000 000000000000000000000000000000000000000 000000000000000000000000000000010000000 000000000000000000000000000000001000000 000000000000000000000000000000000100000 000000010000000000000000000000100000000 000000000000000000000000000000000000000 000000000000000000000000000000000000001 000000000000000000000000000000000000010

Figure 5. SOCIOMATRIX DURING NORMAL PERIOD 


\section{SOCIOMATRIX DURING CRISIS PERIOD}

000000000000000000000000000000000000100 000000000000000000000000000000000000100 000000000000000000000000000000000000100 000000000000000000000000000000000000100 000000000000000000000000000000000000100 000000000000000000000000000000000000100 000000000000000000000000000000000000100 000000000000000000000000000000000000100 000000000000000000000000000000000000100 000000000000000000000000000000000000100 000000000000000000000000000000000000100 000000000000000000000000000000000000100 000000000000000000000000000000000000100 000000000000000000000000000000000000100 000000000000000000000000000000000000100 000000000000000000000000000000000000100 000000000000000000000000000000000000100 000000000000000000000000000000000000100 000000000000000000000000000000000000100 000000000000000000000000000000000000100 000000000000000000000000000000000000100 000000000000000000000000000000000000100 000000000000000000000000000000000000100 000000000000000000000000000000000000100 000000000000000000000000000000000000100 000000000000000000000000000000000000100 000000000000000000000000000000000000100 000000000000000000000000000000000000100 000000000000000000000000000000000000100 000000000000000000000000000000000000100 000000000000000000000000000000000000100 000000000000000000000000000000000000100 000000000000000000000000000000000000100 000000000000000000000000000000000000100 000000000000000000000000000000000000100 000000000000000000000000000000000000100 000000000000000000000000000000000000000 000000000000000000000000000000000000100 000000000000000000000000000000000000100

Figure 6. SOCIOMATRIX DURING CRISIS PERIOD

Degree Centrality
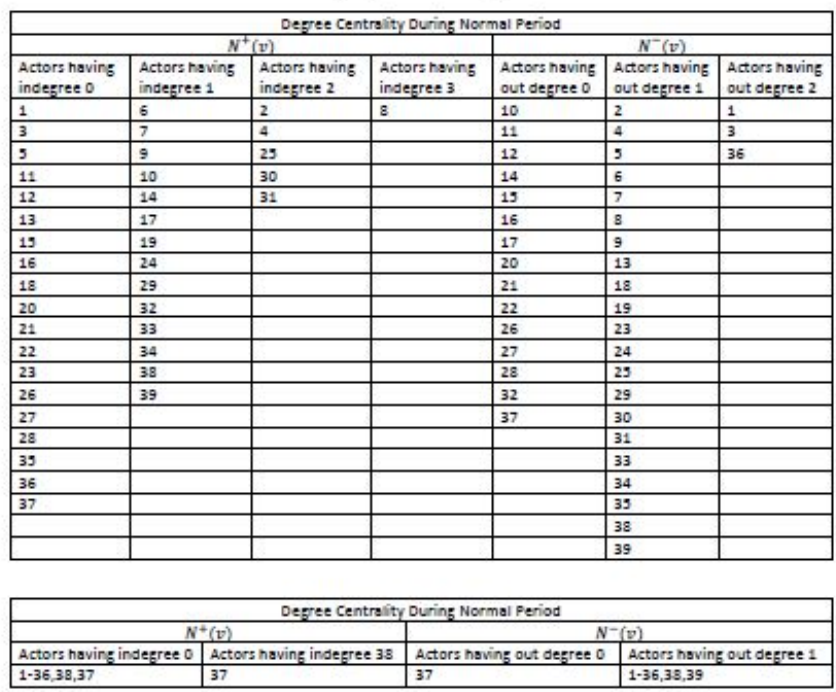

Figure 7. DEGREE CENTRALITY TABLE dently, he seeks the help of samudayam. The matrix clearly indicates that the household of actor 37 is mooppan.

During normal period, nearly half of the families are very weak in society. None of them approaches for companionship, advise and monitory help (even though, they take their on decisions). 36 percentage of families provide mentorship for single actors and 13 percentage being mentor for two families each and 3 percentage are for three families. 38 percentage of actors does not seek any help from others and half of them have a single mentor and 8 percentage are not satisfied for a single mentor, so they seek a second opinion. During crisis period, 97 percentage of families approaches a single mentor, mooppan (actually it is 100 percentage, but the graph under consideration is loop less). High degree centrality is generally considered to be an asset to an actor.

There are 4 reciprocal pairs in our social network during normal period. Only 10 percentage of actors show the tendency to be reciprocated. So the value of the reciprocity is 0.148 during normal period. Due to their social structure, during crisis period, it is obvious that the reciprocity is zero.

Apart from the main theme, it is observed that nearly 21 percentage families are uneducated, the highest educational qualification in lower primary level (1-4 range) is 8 percentage, upper primary level( 5-7) is 10 percentage, high school level (8-10) is 41 percentage and above 10th standard is 20 percentage (maximum higher secondary). 51 percentage are capsule families, 46 percentage families with members 5 to 8 and only 3 percentage are with members more than 8 . There is 23 percentage with youngster households (age less than 40), 49 percentage households having age group 40 - 60, 26 percentage are within $60-80$ and 2 percentage have more than 80.

\section{Conclusion}

The social network field is an interdisciplinary research programme which seeks to predict the structure of relationship among social entities. The field of SNA is broad and growing, and a new methods and approaches are constantly in development. SNA is a group of graph theory based techniques. Graphs are among the most ubiquitous models of both natural and human made structures. That is why it plays the major role in the development of SNA and now being the skeleton.

Even though so many plans were introduced by the Central and State government for the upliftment of the tribal communities, it has shown that there were existing some drawbacks. The proper guidance in the right time can overcome so many problems related to the social matters. So we have decided to study about the interaction among the communities.

Through this study, we tried to develop a tribal community at Elanad, Pazhayannur Panchayath, Kerala, India by 
considering their interaction (advisory help, monitory help and companionship during leisure time) during normal and crisis periods using SNA. We found that most of them does not have a mentor for normal time. It is also noted that they have a unity and integrity at the crisis period. This can be taken as a model to the modern Society(so called). It is very interesting to note that we can treat the human behaviours using graph theoretic tools (like sociomatrix, degree centrality and reciprocity). Primarily it is noted that education does not depend interaction directly. Since the educated groups are youngsters; society give preference to experienced persons than educated. Whenever an external body approaches them for any matter, they aren't ready to disclose their details due to the fear of exploitation. They are not well sound by collecting honey from forest and by agricultural labour. Most of them are in the hands of private financing bodies. We also noted that some of the isolated households posses stronger ties outside the colony.

\section{Acknowledgment}

The authors hereby acknowledge Dr. S. Arumugam, Adjunct Professor, Department of Mathematics, Amrita Vishwa Vidyapeetham, Coimbatore -641112 (Visiting Professor, Department of Computer Science, Liverpool Hope University, Liverpool, UK), (Adjunct Professor, Department of Computer Science, Ball State University, Muncie, USA) for his valuable suggestions and support while preparing this paper.

\section{References}

[1] Brandes, Ulrik, Erlebach, Thomas, Network Analysis, Springer, (2005).

[2] Cheng Wang, Omar Lizardo, David Hachen etal, A Dyadic reciprocity index for repeated interaction networks, ZU064-05-FPR, (2012).

[3] David Knoke, Song Yang, Social Network Analysis (II ), SAGE publications Ltd.

[4] Dr. Rick Davies, The Use of Social Network Analysis Tools in the Evaluation of Social Change Communications, (2009), 11-17.

[5] Durkheim, Emile, The Rules of Sociological Method. The first and most fundamental rule is: Consider social facts as things, (1895), 14.

[6] Helene Barcelo, Reinhard Laubenbacher, Graph Theoretic Tools For Dynamic Network Analysis, 2006.

[7] Helmut K. Anheier, Jurgen Gerhards, Frank P. Romo, Forms of Capital and Social Structure in Cultural Fields: Examining Bourdieu's Social Topography, The American Journal of Sociology, (1995), 859-903.

[8] John Scott, Social Network Analysis(III), SAGE Publications Ltd, 2009.

[9] Kerala Government Senses Report, 2011.

[10] Peter J.Carringtom, John Scott, Staney wasserman, Models and Methods in Social Network Analysis, Cambridge University Press, 2005.
[11] S. Arumugam, B. Haughton, B. Vasanthi and Changan Zhang, Reciprocity in Social Networks-A Case Study in Tamil Nadu India, CS-BIGS, 5(2),(2014), 126-131.

[12] Wasserman S.,Faust K, Social Network Analysis: Methods and Applications, Cambridge, ENG and New York: Cambridge University Press, 1994.

[13] Wouter De Nooy, Fields and networks: Correspondence analysis and social network analysis in the framework of Field Theory, Poetics, 31 (5-6),(2003), 305-327.

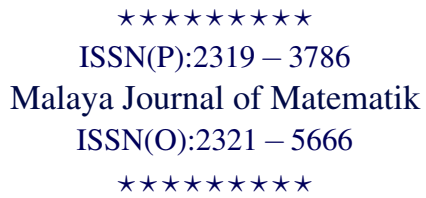

